\title{
Respiratory Distress in the Newborn
}

Suzanne Reuter, MD, ${ }^{*}$ Chuanpit Moser, MD, ${ }^{\dagger}$ Michelle Baack, MD**

*Department of Neonatal-Perinatal Medicine, Sanford School of Medicine-University of South Dakota, Sanford Children's Specialty Clinic, Sioux Falls, SD.

${ }^{\dagger}$ Department of Pediatric Pulmonology, Sanford School of Medicine-University of South Dakota, Sanford Children's Specialty Clinic, Sioux Falls, SD.

${ }^{*}$ Sanford Children's Health Research Center, Sioux Falls, SD.

AUTHOR DISCLOSURES Drs Reuter, Moser and Baack have disclosed no financial relationships relevant to this article. This commentary does not contain information about unapproved/investigative commercial products or devices.

$\begin{array}{ll}\text { ABBREVIATIONS } \\ \text { BPD } & \text { bronchopulmonary dysplasia } \\ \text { CPAP } & \text { continuous positive airway pressure } \\ \text { ECMO } & \begin{array}{l}\text { extracorporal membrane } \\ \text { oxygenation }\end{array} \\ & \text { fraction of inspired oxygen } \\ \text { Fio }_{2} & \text { functional residual capacity } \\ \text { FRC } & \text { group B streptococcus } \\ \text { GBS } & \text { meconium aspiration syndrome } \\ \text { MAS } & \text { meconium-stained amniotic fluid } \\ \text { MSAF } & \text { persistent pulmonary hypertension } \\ \text { PPHN } & \text { of the newborn } \\ \text { PROM } & \text { prolonged rupture of membranes } \\ \text { RDS } & \text { respiratory distress syndrome } \\ \text { TTN } & \text { transient tachypnea of the newborn }\end{array}$

\section{Educational Gap}

Respiratory distress is common, affecting up to $7 \%$ of all term newborns, (1) and is increasingly common in even modest prematurity. Preventive and therapeutic measures for some of the most common underlying causes are well studied and when implemented can reduce the burden of disease. (2)(3)(4)(5)(6)(7)(8) Failure to readily recognize symptoms and treat the underlying cause of respiratory distress in the newborn can lead to short- and long-term complications, including chronic lung disease, respiratory failure, and even death.

\section{Objectives After completing this article, the reader should be able to:}

1. Use a physiologic approach to understand and differentially diagnose the most common causes of respiratory distress in the newborn infant.

2. Distinguish pulmonary disease from airway, cardiovascular, and other systemic causes of respiratory distress in the newborn.

3. Appreciate the risks associated with late preterm (34-36 weeks' gestation) and early term (37-38 weeks' gestation) deliveries, especially by caesarean section.

4. Recognize clinical symptoms and radiographic patterns that reflect transient tachypnea of the newborn (TTN), neonatal pneumonia, respiratory distress syndrome (RDS), and meconium aspiration syndrome (MAS).

5. Identify the short- and long-term complications associated with common neonatal respiratory disorders, including pneumothorax, persistent pulmonary hypertension of the newborn, and chronic lung disease.

6. Understand management strategies for TTN, pneumonia, RDS, and MAS.

7. Implement up-to-date recommendations for the prevention of neonatal pneumonia, RDS, and MAS. 


\section{INTRODUCTION}

Respiratory distress is one of the most common reasons an infant is admitted to the neonatal intensive care unit. (I) Fifteen percent of term infants and $29 \%$ of late preterm infants admitted to the neonatal intensive care unit develop significant respiratory morbidity; this is even higher for infants born before 34 weeks' gestation. (2) Certain risk factors increase the likelihood of neonatal respiratory disease. These factors include prematurity, meconium-stained amniotic fluid (MSAF), caesarian section delivery, gestational diabetes, maternal chorioamnionitis, or prenatal ultrasonographic findings, such as oligohydramnios or structural lung abnormalities. (2)(9)(IO)(II)(I2)(I3)(I4) However, predicting which infants will become symptomatic is not always possible before birth. Regardless of the cause, if not recognized and managed quickly, respiratory distress can escalate to respiratory failure and cardiopulmonary arrest. Therefore, it is imperative that any health care practitioner caring for newborn infants can readily recognize the signs and symptoms of respiratory distress, differentiate various causes, and initiate management strategies to prevent significant complications or death.

\section{DEFINITION, SIGNS, SYMPTOMS}

Respiratory distress in the newborn is recognized as one or more signs of increased work of breathing, such as tachypnea, nasal flaring, chest retractions, or grunting. (I)(I5) Normally, the newborn's respiratory rate is 30 to 60 breaths per minute. Tachypnea is defined as a respiratory rate greater than 60 breaths per minute. (15) Tachypnea is a compensatory mechanism for hypercarbia, hypoxemia, or acidosis (both metabolic and respiratory), (I6) making it a common but nonspecific finding in a large variety of respiratory, cardiovascular, metabolic, or systemic diseases. Pulmonary disease may incite tachypnea, especially in neonates. The natural elastic property of the lungs is to deflate. When balanced by the outward recoil of the chest wall, functional residual capacity (FRC) occurs at the end of expiration to prevent alveoli from collapsing. The newborn chest wall, composed primarily of cartilage, is more pliable, predisposing neonatal lungs to pulmonary atelectasis and decreased FRC. (I6)(I7)(I8) Pulmonary compliance refers to a given change in volume ( $\Delta$ Volume) for every given change in pressure ( $\Delta$ Pressure), essentially the ability of the alveoli to fill with air under a set pressure. If lung compliance is decreased, such as with transient tachypnea of the newborn (TTN), respiratory distress syndrome (RDS), pneumonia, or pulmonary edema, there is a decrease in tidal volume. To achieve sufficient minute ventilation, the respiratory rate must increase. Hypoxemia further increases tachypnea. (I6)(I8) Therefore, affected newborns present with marked tachypnea. Because tachypnea is a nonspecific symptom, additional clinical findings aid in narrowing the cause to a respiratory disorder.

Increased work of breathing results from mismatched pulmonary mechanics from increased airway resistance ( $\Delta$ Pressure/Volumetric Flow), decreased lung compliance ( $\Delta$ Volume/ $\Delta$ Pressure), or both. Airway resistance increases when there is obstruction of air flow. The critical importance of airway radius is indicated in the equation $\mathrm{R}=\mathrm{V}(81 \eta / \pi \mathrm{r}$ (4)), where $\mathrm{R}$ is resistance, $\mathrm{V}$ is flow, $\mathrm{l}$ is length, $\eta$ is viscosity, and $r$ is radius. (I9) If the airway radius is halved, resistance increases I6-fold. Nasal flaring is a compensatory symptom that increases upper airway diameter and reduces resistance and work of breathing. Retractions, evident by the use of accessory muscles in the neck, rib cage, sternum, or abdomen, occur when lung compliance is poor or airway resistance is high. Noisy breathing may indicate increased airway resistance, and the type of noise auscultated may help localize airway obstruction (Table I). Stertor is a sonorous snoring sound heard over extrathoracic airways that indicates nasopharyngeal obstruction. Stridor is a high-pitched, monophonic breath sound that indicates obstruction at the larynx, glottis, or subglottic area. Wheezing may also be high pitched but is typically polyphonic, is heard on expiration, and indicates tracheobronchial obstruction. Grunting is an expiratory sound caused by sudden closure of the glottis during expiration in an attempt to maintain FRC and prevent alveolar atelectasis. Because lung compliance is worse at very low or very high FRC, achieving and maintaining physiologic FRC is essential in the management of respiratory disorders with poor compliance, such as RDS or TTN. On the other end of the spectrum, meconium aspiration syndrome (MAS) is an example of lower airway obstruction with air trapping. These newborns often have high lung volumes, which adversely affects their lung compliance. Regardless of the cause, it is vital to recognize symptoms and act quickly. If the newborn cannot sustain the extra work of breathing to meet its respiratory needs, respiratory failure follows. This failure may manifest as impaired oxygenation (cyanosis) or ventilation (respiratory acidosis). Without prompt intervention, respiratory arrest is imminent.

\section{PATHOGENESIS}

The causes of respiratory distress in a newborn are diverse and multisystemic. Pulmonary causes may be related to alterations during normal lung development or transition to extrauterine life. Normal lung development occurs in 5 
TABLE 1. Noisy Breathing Characteristics in Term Infants

\begin{tabular}{|c|c|c|}
\hline TYPE & DEFINITION & CAUSES \\
\hline Stertor & $\begin{array}{l}\text { Sonorous snoring sound, mid-pitched, monophonic, may } \\
\text { transmit throughout airways, heard loudest with } \\
\text { stethoscope near mouth and nose }\end{array}$ & $\begin{array}{l}\text { Nasopharyngeal obstruction-nasal or airway secretions, } \\
\text { congestion, choanal stenosis, enlarged or redundant upper } \\
\text { airway tissue or tongue }\end{array}$ \\
\hline Stridor & $\begin{array}{l}\text { Musical, monophonic, audible breath sound. Typically } \\
\text { high-pitched. Types: Inspiratory (above the vocal cords), } \\
\text { biphasic (at the glottis or subglottis), or expiratory } \\
\text { (lower trachea) }\end{array}$ & $\begin{array}{l}\text { Laryngeal obstruction-laryngomalacia, vocal cord paralysis, } \\
\text { subglottic stenosis, vascular ring, papillomatosis, foreign } \\
\text { body }\end{array}$ \\
\hline Wheezing & $\begin{array}{l}\text { High-pitched, whistling sound, typically expiratory, } \\
\text { polyphonic, loudest in chest }\end{array}$ & Lower airway obstruction-MAS, bronchiolitis, pneumonia \\
\hline Grunting & $\begin{array}{l}\text { Low- or mid-pitched, expiratory sound caused by } \\
\text { sudden closure of the glottis during expiration } \\
\text { in an attempt to maintain FRC }\end{array}$ & $\begin{array}{l}\text { Compensatory symptom for poor pulmonary compliance- } \\
\text { TNN, RDS, pneumonia, atelectasis, congenital lung } \\
\text { malformation or hypoplasia, pleural effusion, pneumothorax }\end{array}$ \\
\hline
\end{tabular}

$F R C=$ functional residual capacity; $M A S=$ meconium aspiration syndrome; $R D S=$ respiratory distress syndrome; $T$ N $=$ transient tachypnea of the newborn.

phases (20) (Table 2). Respiratory disease may result from developmental abnormalities that occur before or after birth. Early developmental malformations include tracheoesophageal fistula, bronchopulmonary sequestration (abnormal mass of pulmonary tissue not connected to the tracheobronchial tree), and bronchogenic cysts (abnormal branching of the tracheobronchial tree). Later in gestation, parenchymal lung malformations, including congenital cystic adenomatoid malformation or pulmonary hypoplasia from congenital diaphragmatic hernia or severe oligohydramnios, may develop. More common respiratory diseases, such as TTN, RDS, neonatal pneumonia, MAS, and persistent pulmonary hypertension of the newborn (PPHN), result from complications during the prenatal to postnatal transition period. Although mature alveoli are present at 36 weeks' gestation, a great deal of alveolar septation and microvascular maturation occur postnatally. The lungs are not fully developed until ages 2 to 5 years. (20)(2I) Therefore, developmental lung disease can also occur after birth. Bronchopulmonary dysplasia (BPD), for example, is a significant lung disease that complicates prematurity due to arrested alveolarization in developing lungs exposed to mechanical ventilation, oxygen, and other inflammatory mediators before normal development is complete. As defined by an ongoing oxygen requirement at 36 weeks' adjusted gestational age, BPD affects up to $32 \%$ of premature infants and $50 \%$ of very low-birth-weight infants. (22)

\section{DIFFERENTIAL DIAGNOSIS}

The underlying cause of respiratory distress in a newborn varies and does not always lie within the lungs (I5) (Table 3).
Thus, after initial resuscitation and stabilization, it is important to use a detailed history, physical examination, and radiographic and laboratory findings to determine a more specific diagnosis and appropriately tailor management. A thorough history may guide in identifying risk factors associated with common causes of neonatal respiratory distress (Table 4). A detailed physical examination should focus beyond the lungs to identify nonpulmonary causes, such as airway obstruction, abnormalities of the chest wall, cardiovascular disease, or neuromuscular disease, that may initially present as respiratory distress in a newborn. Radiographic findings can identify diaphragmatic paralysis, congenital pulmonary malformations, and intrathoracic spaceoccupying lesions, such as pneumothorax, mediastinal mass, and congenital diaphragmatic hernia, that can compromise lung expansion. Significant tachypnea without increased work of breathing should prompt additional laboratory investigation to identify metabolic acidosis or sepsis. Hypoglycemia, hypomagnesemia, and hematologic abnormalities may result in a depressed ventilatory drive or impaired oxygen transport to the peripheral tissues, so laboratory evaluation should also be considered with these clinical findings. Hypermagnesemia may contribute to respiratory distress and affect a newborn's capacity to respond to resuscitation due to hypotonia and a depressed respiratory drive or even apnea.

Cardiovascular disease may be difficult to distinguish from pulmonary causes of respiratory distress (Table 5). Most congenital heart defects present with cyanosis, tachypnea, or respiratory distress from cardiac failure. Timing may be an important clue to differentiation because very few congenital heart defects present immediately after birth; 
TABLE 2. Developmental Stages of Lung Development and Respiratory Disease Pathogenesis

\begin{tabular}{|c|c|c|c|c|c|}
\hline $\begin{array}{l}\text { DEVELOPMENTAL } \\
\text { STAGE }\end{array}$ & EMBRYONIC & PSEUDOGLANDULAR & CANALICULAR & TERMINAL SAC & ALVEOLAR \\
\hline Gestation & 0-6 weeks & 7-16 weeks & 17-24 weeks & 25-36 weeks & $>37$ weeks \\
\hline $\begin{array}{l}\text { Structural } \\
\text { morphogenesis }\end{array}$ & Trachea, bronchi & $\begin{array}{l}\text { Bronchioles, terminal } \\
\text { bronchioles, lung } \\
\text { circulation }\end{array}$ & $\begin{array}{l}\text { Respiratory bronchioles, } \\
\text { primitive alveoli }\end{array}$ & $\begin{array}{l}\text { Alveolar ducts, thin- } \\
\text { walled alveolar sacs, } \\
\text { increasing } \\
\text { functional } \\
\text { type } 2 \text { cells }{ }^{\mathrm{a}}\end{array}$ & $\begin{array}{l}\text { Definitive alveoli } \\
\text { and mature } \\
\text { type } 2 \text { cells }^{a}\end{array}$ \\
\hline $\begin{array}{l}\text { Disease } \\
\quad \text { manifestation }\end{array}$ & $\begin{array}{l}\text { Tracheoesophageal } \\
\text { fistula, pulmonary } \\
\text { sequestration }\end{array}$ & $\begin{array}{l}\text { Bronchogenic cyst, } \\
\text { congenital } \\
\text { diaphragmatic } \\
\text { hernia, } \\
\text { congenital cystic } \\
\text { adenomatoid } \\
\text { malformation }\end{array}$ & $\begin{array}{l}\text { Pulmonary hypoplasia, } \\
\text { RDS, BPD, alveolar } \\
\text { capillary dysplasia }\end{array}$ & RDS, BPD & $\begin{array}{l}\text { TTN, MAS, } \\
\text { neonatal } \\
\text { pneumonia, } \\
\text { PPHN }\end{array}$ \\
\hline
\end{tabular}

$B P D=$ bronchopulmonary dysplasia; $M A S=$ meconium aspiration syndrome; $P P H N=$ persistent pulmonary hypertension of the newborn; $R D S=$ respiratory distress syndrome; TTN=transient tachypnea of the newborn;

${ }^{a}$ Type 2 pneumocytes are surfactant-producing cells

more often they present several hours to days after delivery as the ductus arteriosus closes. (2) Table 5 aids in this differentiation.

Pulmonary hypertension should be considered in any infant with respiratory distress and cyanosis. This condition results when there is a failure to transition from in utero to postnatal pulmonary circulation after delivery. Pulmonary vascular resistance remains high, resulting in cyanosis from impaired pulmonary blood flow and right-to-left shunting of blood across the foramen ovale and ductus arteriosus. Shunting further contributes to systemic hypoxemia and metabolic academia-both of which contribute to ongoing increased pulmonary vascular resistance. PPHN may be primary or secondary to respiratory disease, particularly congenital diaphragmatic hernia, MAS, or RDS. When PPHN occurs without concurrent pulmonary disease, differentiating from cyanotic heart disease is difficult. The response to ventilation with I00\% oxygen (hyperoxia test) can help distinguish the 2 conditions. In some neonates with PPHN, the $\mathrm{PaO}_{2}$ will increase to above $100 \mathrm{~mm} \mathrm{Hg}$, whereas it will not increase above $45 \mathrm{~mm} \mathrm{Hg}$ in infants with cyanotic heart defects that have circulatory mixing. (5)(23)

\section{COMMON CASE SCENARIOS}

Four case scenarios are highlighted to help in identifying the most common causes of respiratory distress in the newborn followed by discussion about the pathophysiology, risk factors, prevention, and management strategies for each disorder.

\section{Case 1}

A 3.2-kg female infant is delivered by caesarean section at 38 weeks' gestational age without a trial of labor. Her Apgar scores are 9 and 9 at $\mathrm{I}$ and 5 minutes, respectively. She develops tachypnea and subcostal retractions with nasal flaring at I hour of life. Temperature is $97.9^{\circ} \mathrm{F}\left(36.6^{\circ} \mathrm{C}\right)$, pulse is $\mathrm{I}_{5}$ beats per minute, and respiratory rate is 74 breaths per minute. Aside from increased work of breathing, her physical examination findings are normal. The chest radiograph is shown in Figure I. She requires supplemental oxygen via nasal cannula with a fraction of inspired oxygen $\left(\mathrm{FiO}_{2}\right)$ of 0.3 for 36 hours. She then weans to room air. Her respiratory rate is 35 breaths per minute, and she has no increased work of breathing.

\section{Transient Tachypnea of the Newborn}

TTN, also known as retained fetal lung fluid syndrome, presents with early respiratory distress in term and latepreterm infants. TTN is a frequent cause of respiratory distress in newborns and is caused by impaired fetal lung fluid clearance. Normally in utero, the fetal airspaces and air sacs are fluid filled. For effective gas exchange to occur after birth, this fluid must be cleared from the alveolar airspaces. Late in gestation and before birth, the chloride and fluid-secreting channels in the lung epithelium are reversed so that fluid absorption predominates and fluid is removed from the lungs. This process is enhanced by labor, so that delivery before labor onset increases the risk of retained fetal lung fluid. (20) Factors that increase the clearance of lung fluid include antenatal corticosteroids, fetal thorax compression 
TABLE 3. Differential Diagnosis of Respiratory Distress in the Newborn

\begin{abstract}
Airway
Nasal obstruction, choanal atresia, micrognathia, Pierre Robin sequence, macroglossia, congenital high airway obstruction syndrome, including laryngeal or tracheal atresia, subglottic stenosis, laryngeal cyst or laryngeal web, vocal cord paralysis, subglottic stenosis, airway hemangiomas or papillomas, laryngomalacia, tracheobronchomalacia, tracheoesophageal fistula vascular rings, and external compression from a neck mass
\end{abstract}

\section{Pulmonary}

$\mathrm{RDS}^{\mathrm{a}}{ }^{\mathrm{T}} \mathrm{TN},{ }^{\mathrm{a}} \mathrm{MAS},{ }^{\mathrm{a}}$ neonatal pneumonia, ${ }^{\mathrm{a}}$ pneumothorax, ${ }^{\mathrm{a}} \mathrm{PPHN},{ }^{a}$ pleural effusion (congenital chylothorax), pulmonary hemorrhage, bronchopulmonary sequestration, bronchogenic cyst, congenital cystic adenomatoid malformation or congenital pulmonary airway malformation, pulmonary hypoplasia, congenital lobar emphysema, pulmonary alveolar proteinosis, alveolar capillary dysplasia, congenital pulmonary lymphangiectasis, and surfactant protein deficiency

\section{Cardiovascular}

Cyanotic and select acyanotic congenital heart defects, ${ }^{a}$ neonatal cardiomyopathy, pericardial effusion or cardiac tamponade, fetal arrhythmia with compromised cardiac function, and high-output cardiac failure

\section{Thoracic}

Pneumomediastinum, chest wall deformities, mass, skeletal dysplasia, and diaphragmatic hernia or paralysis

\begin{tabular}{|c|}
\hline Neuromuscular \\
\hline $\begin{array}{l}\text { Central nervous system injury (birth trauma or hemorrhage), }{ }^{a} \\
\text { hypoxic-ischemic encephalopathy, }{ }^{a} \text { cerebral malformations, } \\
\text { chromosomal abnormalities, medication (neonatal or maternal } \\
\text { sedation, antidepressants, or magnesium), congenital TORCH } \\
\text { infections, meningitis, seizure disorder, obstructed } \\
\text { hydrocephalus, arthrogryposis, congenital myotonic dystrophy, } \\
\text { neonatal myasthenia gravis, spinal muscular atrophy, congenital } \\
\text { myopathies, and spinal cord injury }\end{array}$ \\
\hline Other \\
\hline $\begin{array}{l}\text { Sepsis, }{ }^{a} \text { hypoglycemia, }{ }^{a} \text { metabolic acidosis, }{ }^{a} \text { hypothermia or } \\
\text { hyperthermia, hydrops fetalis, inborn error of metabolism, } \\
\text { hypermagnesemia, hyponatremia or hypernatremia, severe } \\
\text { hemolytic disease, anemia, and polycythemia }\end{array}$ \\
\hline
\end{tabular}

$B P D=$ bronchopulmonary dysplasia; $M A S=$ meconium aspiration syndrome; $P P H N=$ persistent pulmonary hypertension of the newborn; $R D S=$ respiratory distress syndrome; $T \mathrm{~T}=$ transient tachypnea of the newborn.

${ }^{a}$ Relatively common causes of respiratory distress in the newborn.

with uterine contractions, and a release of fetal adrenaline in labor, which enhances uptake of lung fluids. (24)

Infants with TTN usually present with tachypnea and increased work of breathing, which persists for 24 to 72 hours. Chest radiographs reveal excess diffuse parenchymal infiltrates due to fluid in the interstitium, fluid in the interlobar fissure, and occasionally pleural effusions (Figure I). Management is supportive. Infants may require supplemental oxygen, and frequently the distending forces of continuous positive airway pressure (CPAP) are necessary to assist in maintaining alveolar integrity and driving fluid into circulation. Blood gases often reveal a mild respiratory acidosis and hypoxemia. The course of TTN is self-limited and does not usually require mechanical ventilation.

Preventive measures may include avoiding elective caesarean section before the onset of labor in infants younger than 39 weeks' gestation. This is because the most common risk factors for TTN include delivery before 39 weeks' gestation, (I)(2)(3)(9)(25)(26) precipitous delivery, fetal distress, maternal sedation, and maternal diabetes. Although it is well known that premature infants have a higher risk of respiratory problems, the consequences of early-term delivery (37-38 weeks' gestation) are underrecognized. Earlyterm infants have an increased risk of requiring respiratory support, mechanical ventilation, and neonatal service; delivery by caesarean section in this population is common and further increases risk. (25) In addition, a single course of antenatal glucocorticoids ( 2 doses of betamethasone) at least 48 hours before an elective term caesarean delivery decreases respiratory morbidity among infants. (27) On the basis of multiple cohort studies and expert opinion, we recommend a careful consideration about elective delivery before spontaneous onset of labor at less than 39 weeks' gestation and encourage pediatricians to be aware of the increased risk of respiratory morbidity in late preterm and early-term newborns. (I)(2)(3)(9)(25)(26)

\section{Case 2}

A 2.9 -kg male infant is born by vaginal delivery at 39 weeks' gestational age after rupture of membranes for 22 hours. Apgar scores are 8 and 8 at I and 5 minutes, respectively. He requires an $\mathrm{FiO}_{2}$ of 0.4 in the delivery room. He is tachypneic and has acrocyanosis. There are coarse rales noted bilaterally. Temperature is $98.6^{\circ} \mathrm{F}\left(37^{\circ} \mathrm{C}\right)$, pulse is 144 beats per minute, and respiratory rate is $6_{5}$ breaths per minute. Despite being given CPAP, his grunting and tachypnea worsen, and he requires intubation and ventilation for progressive increased work of breathing, respiratory acidosis, and oxygen requirement during the next 6 hours. The chest radiograph is shown in Figure I.

\section{Neonatal Pneumonia}

Respiratory infections in the newborn may be bacterial, viral, fungal, spirochetal, or protozoan in origin. Infants may acquire pneumonia transplacentally, through infected amniotic fluid, via colonization at the time of birth, or nosocomially. (20) Perinatal pneumonia is the most common form of neonatal pneumonia and is acquired at birth. 
TABLE 4. Perinatal History Associated With Common Respiratory

Diseases in the Newborn Infant

\begin{tabular}{ll} 
RESPIRATORY DISEASE & RISK FACTORS \\
\hline TTN & $\begin{array}{c}\text { Caesarian section, precipitous delivery, late preterm or early term, maternal sedation or medication, fetal } \\
\text { distress, gestational diabetes }\end{array}$ \\
\hline Neonatal pneumonia & $\begin{array}{c}\text { Maternal group B streptococcus carrier, chorioamnionitis, maternal fever, PROM, prematurity, perinatal } \\
\text { depression }\end{array}$ \\
\hline MAS & Prematurity, gestational diabetes, male infant, multiple gestation \\
\hline Pulmonary hypoplasia & MSAF, postterm gestation, fetal distress or perinatal depression, African American ethnicity \\
\hline
\end{tabular}

$M A S=$ meconium aspiration syndrome; $M S A F=$ meconium-stained amniotic fluid; $P R O M=$ prolonged rupture of membranes; RDS=respiratory distress syndrome; $T \mathrm{~T}=$ transient tachypnea of the newborn.

Group B streptococcus (GBS) is the most common organism that affects term infants. (28)(29) Congenital pneumonia occurs when the causative organism is passed transplacentally to the fetus. The most common pathogens are rubella, cytomegalovirus, adenovirus, enteroviruses, mumps, Toxoplasma gondii, Treponema pallidum, Mycobacterium tuberculosis, Listeria monocytogenes, varicella zoster, and human immunodeficiency virus. (30) Immaturity of the infant's immune system and the pulmonary anatomical and physiologic features make the newborn at higher risk of infection. The underdeveloped respiratory cilia and the decreased number of pulmonary macrophages result in decreased clearance of pathogens from the respiratory system. Newborns also have diminished cellular and humoral immune function, which is even more pronounced in the premature infant. (28)

Risk factors for perinatal pneumonia include prolonged rupture of membranes (PROM), maternal infection, and

\section{TABLE 5. Differentiation of Cyanotic Heart Disease From Pulmonary Disease Among Infants in Respiratory Distress ${ }^{\mathrm{a}}$}

\begin{tabular}{|c|c|c|}
\hline VARIABLE & CYANOTIC HEART DISEASE & PULMONARY DISEASE \\
\hline History & $\begin{array}{l}\text { Previous sibling with congenital heart disease } \\
\text { Diagnosis of congenital heart disease by prenatal } \\
\text { ultrasonography }\end{array}$ & $\begin{array}{l}\text { Maternal fever } \\
\text { MSAF } \\
\text { Preterm delivery }\end{array}$ \\
\hline Physical examination & $\begin{array}{l}\text { Cyanosis } \\
\text { Gallop rhythm or murmur } \\
\text { Single second heart sound } \\
\text { Large liver } \\
\text { Mild respiratory distress }\end{array}$ & $\begin{array}{l}\text { Cyanosis } \\
\text { Severe retractions } \\
\text { Split second heart sound } \\
\text { Temperature instability }\end{array}$ \\
\hline Chest radiograph & $\begin{array}{l}\text { Increased heart size } \\
\text { Decreased pulmonary vascularity (except in } \\
\text { transposition of the great vessels or total } \\
\text { anomalous pulmonary venous return) }\end{array}$ & $\begin{array}{l}\text { Normal heart size } \\
\text { Abnormal pulmonary parenchyma, such as total } \\
\text { whiteout or patches of consolidation in pneumonia, } \\
\text { fluid in the fissures in TTN or ground glass } \\
\text { appearance in RDS }\end{array}$ \\
\hline Arterial blood gas & $\begin{array}{l}\text { Normal or decreased } \mathrm{PacO}_{2} \\
\text { Decreased } \mathrm{PaO}_{2}\end{array}$ & $\begin{array}{l}\text { Increased } \mathrm{PaCO}_{2} \\
\text { Decreased } \mathrm{PaO}_{2}\end{array}$ \\
\hline Hyperoxia test & $\mathrm{PaO}_{2}<150 \mathrm{~mm} \mathrm{Hg}$ & $\mathrm{PaO}_{2}>150 \mathrm{~mm} \mathrm{Hg}$ (except in severe PPHN) \\
\hline Echocardiography & Abnormal heart or vessels & Normal heart and vessels \\
\hline
\end{tabular}

MSAF=meconium-stained amniotic fluid; $P P H N=$ persistent pulmonary hypertension of the newborn; RDS=respiratory distress syndrome; $T$ TN=transient tachypnea of the newborn.

${ }^{a}$ Reproduced with permission from Aly et al. (23) Copyright 2014 by the American Academy of Pediatrics. 
Figure 1. Case 1: Transient tachypnea of the newborn is characterized by streaky, pulmonary interstitial markings and fluid in the fissure apparent on chest radiograph. Case 2: Neonatal pneumonia with bilateral opacities, air bronchograms, and pleural effusions is apparent. Case 3: Respiratory distress syndrome is characterized by diffuse, bilateral, ground glass fields with air bronchograms secondary to diffuse atelectasis. Case 4: Meconium aspiration syndrome causes a chemical pneumonitis, partial airway obstruction, and a localized surfactant inactivation that leads to areas of hyperinflation mixed with diffuse, patchy infiltrates radiographically.
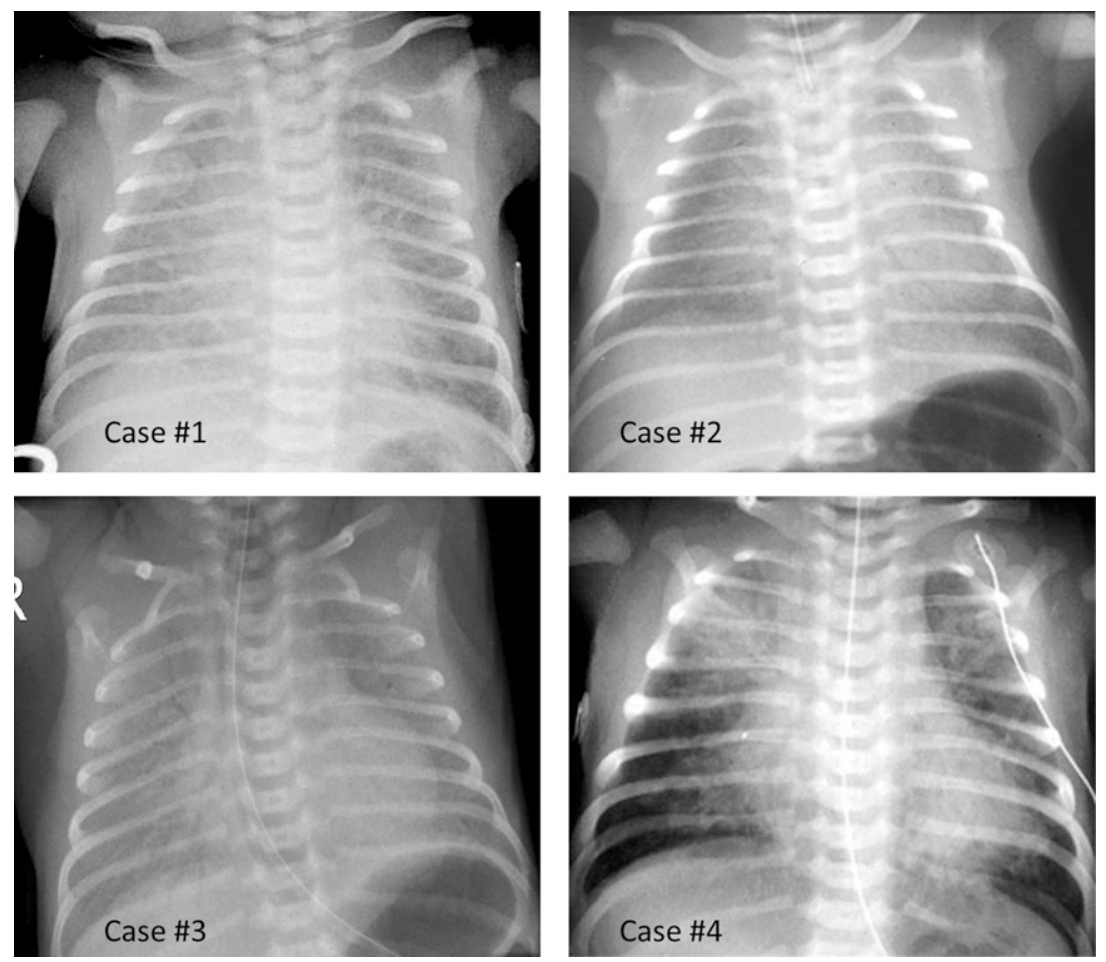

prematurity. (I) Infants present with increased work of breathing and oxygen requirement. Chest radiography often reveals diffuse parenchymal infiltrates with air bronchograms or lobar consolidation. Pleural effusions may also be seen. In contrast to older infants and children, neonatal pneumonia is part of a generalized sepsis illness; thus, obtaining blood and cerebrospinal fluid cultures and initiating broad-spectrum antibiotic therapy is recommended for any symptomatic infant. (3I)(32)

In the newborn with early-onset pneumonia or sepsis, a combination of penicillin and an aminoglycoside are the preferred initial treatment. (3I) For infants who have been hospitalized in a neonatal intensive care unit for more than 4 days, organisms such as methicillin-resistant Staphylococcus aureus and Staphylococcus epidermidis require vancomycin therapy. Infants who develop pneumonia in the nursery or at home are likely to have infections caused by respiratory viruses (adenovirus, respiratory syncytial virus, and influenza virus), gram-positive bacteria (streptococcal species and $S$ aureus), and gram-negative enteric bacteria (Klebsiella, Proteus, Pseudomonas aeruginosa, Serratia marcescens, and Escherichia coli). (30) Infants with pneumonia caused by Chlamydia trachomatis present later in the newborn period (4-I2 weeks of age) with a staccato cough but no wheezing or fever. (33) Chlamydial conjunctivitis may also be present (5 to I4 days after birth). Chest radiography reveals diffuse bilateral infiltrates, and a complete blood cell count with a differential reveals eosinophilia. Treatment of chlamydial pneumonia or conjunctivitis (even without pneumonia) requires systemic macrolide antibiotic therapy and ophthalmologic follow-up. Regardless of the causal organism, newborns with pneumonia require supportive care in addition to antibiotics. Many infants will require not only supplemental oxygen but also CPAP and mechanical ventilation. Other supportive measures include intravenous nutrition and vasopressors for cardiovascular support. PPHN is a common complication of neonatal pneumonia.

On the basis of strong evidence, prevention of neonatal pneumonia and its complications focuses on maternal GBS screening, intrapartum antibiotic prophylaxis, and appropriate follow-up of newborns at high risk after delivery. (4) (3I)(32)(34) Anyone caring for newborns should be able to recognize at-risk infants and whether appropriate intrapartum antibiotic prophylaxis has been administered. They must also know which infants require additional screening, observation, and antibiotic initiation after birth. Guidelines have been established by the Centers for Disease Control and Prevention and endorsed by the American Academy of Pediatrics and the American College of Obstetrics and Gynecology for best practice management of at-risk infants. (4) Infants who require additional attention include those born to mothers who are GBS carriers (culture or polymerase 
chain reaction positive), those with a history of GBS bacteruria, those affected by GBS or with an unknown GBS status but who were delivered at less than 37 weeks' gestation, those with PROM of I8 hours or long, or those with intrapartum fever ( $\left.\geq \mathrm{IO0} \cdot 4^{\circ} \mathrm{F}\left[38^{\circ} \mathrm{C}\right]\right)$. (4)(3I) The preferred intrapartum antibiotic for these situations is intravenous penicillin (5 million units followed by 2.5 million to 3.0 million units every 4 hours) administered at least 4 hours before delivery; cefazolin may be used for penicillin-allergic women who are at low risk for anaphylaxis. (4)(3I) For severely penicillinallergic women, clindamycin culture sensitivity should be performed, and if mother's strain is sensitive (75\% of cases), clindamycin should be used. Vancomycin is reserved for severely allergic women with resistant strains. (4)(3I) In addition to intrapartum antibiotic prophylaxis, promising GBS vaccines are in clinical trials (35) and may be widely accepted by patients (36) but are not yet ready for general use.

Since widespread implementation of maternal GBS screening and intrapartum antibiotic prophylaxis administration, the incidence of early-onset GBS infection has decreased from I. 8 cases per I, 000 to 0.3 case per I,, 00 live births. (3I)(32) However, cases and deaths continue to occur with GBS as the leading offender. (3I)(34)(35) Most of the term infants affected are born to mothers without or with an unknown GBS status but who had PROM or fever and did not receive antibiotic administration during labor. (34) Others are born to women who received inadequate prophylaxis $(<4$ hours before delivery or macrolide antibiotic use). (3I) Many missed opportunities for prevention increase the burden of disease. (29)

Thus, it is imperative to appropriately manage any newborn with the aforementioned risk factors cautiously after birth. According to updated 2010 guidelines, any infant who develops signs or symptoms of illness requires a full diagnostic evaluation (including blood and spinal fluid cultures) and antibiotic initiation. (4)(3I)(32) If maternal chorioamnionitis is suspected but the infant has no signs or symptoms of disease, a limited evaluation (blood culture and complete blood cell count), along with antibiotic therapy initiation for at least 48 hours, is recommended. (4)(3I)(32) Asymptomatic, at-risk infants, who did not receive adequate antibiotic prophylaxis, require a limited evaluation and observation for 48 hours, but antibiotic initiation is not necessary unless clinical suspicion arises. (4)(3I)(32) Asymptomatic, atrisk infants who received adequate intrapartum antibiotic prophylaxis should be observed for 48 hours. Adherence to these guidelines will decrease the incidence of neonatal pneumonia and allow for early detection and treatment that may prevent life-threatening complications, such as PPHN or death.
Case 3

A 1.5 -kg male is delivered via vaginal delivery because of preterm labor at 33 weeks' gestation. Apgar scores are 7 and 8 at $\mathrm{I}$ and 5 minutes, respectively. The infant is cyanotic and requires CPAP immediately after delivery. He has subcostal retractions, grunting, and nasal flaring. Auscultation reveals decreased air entry in the lung fields throughout. Temperature is $98.2^{\circ} \mathrm{F}\left(36.8^{\circ} \mathrm{C}\right)$, pulse is 175 beats per minute, and respiratory rate is 70 breaths per minute. He requires an $\mathrm{FiO}_{2}$ of 0.4 . His chest radiograph is shown in Figure I.

\section{Respiratory Distress Syndrome}

RDS, also known as hyaline membrane disease, is a common cause of respiratory disease in the premature infant. RDS is also seen in infants whose mothers have diabetes in pregnancy. RDS is caused by a deficiency of alveolar surfactant, which increases surface tension in alveoli, resulting in microatelectasis and low lung volumes. Surfactant deficiency appears as diffuse fine granular infiltrates on radiograph (Figure I). Pulmonary edema plays a central role in the pathogenesis of RDS and contributes to the development of air bronchograms. Excess lung fluid is attributed to epithelial injury in the airways, decreased concentration of sodium-absorbing channels in the lung epithelium, and a relative oliguria in the first 2 days after birth in premature infants. (37) Infants typically improve on onset of diuresis by the fourth day after birth.

Infants with RDS typically present within the first several hours of life, often immediately after delivery. Clinically, infants have marked respiratory distress with tachypnea, nasal flaring, grunting, and subcostal, intercostal, and/or suprasternal retractions. Grunting occurs when an infant attempts to maintain an adequate FRC in the face of poorly compliant lungs by partial glottic closure. As the infant prolongs the expiratory phase against this partially closed glottis, there is a prolonged and increased residual volume that maintains the airway opening and also an audible expiratory sound. Infants with RDS have cyanosis and require supplemental oxygen. Mild cases of RDS may respond to the distending pressures of CPAP, but more severe cases require endotracheal intubation and administration of exogenous surfactant into the lungs. Currently, there are no universal guidelines that dictate if and when to administer exogenous surfactant. Some institutions advocate administration of prophylactic surfactant in the first 2 hours of life for all premature infants younger than 30 weeks' gestation. Others begin with noninvasive ventilation (CPAP) and reserve intubation and surfactant administration only for infants who require more than $35 \%$ to $45 \%$ oxygen concentration to maintain an arterial $\mathrm{PaO}_{2}$ greater 
than $50 \mathrm{~mm} \mathrm{Hg}$. In determining a management strategy, it is important to consider the administration of antenatal corticosteroids, the clinical presentation, radiographic findings, and the infant's oxygen requirements. (38)

The course of RDS is self-limited and typically improves by age 3 to 4 days in correlation with the aforementioned diuresis phase and as the infant begins to produce endogenous surfactant. (20) Use of mechanical ventilation before this is supportive and should proceed with caution to avoid ventilator-induced lung injury. Infants who do not improve with surfactant administration should be evaluated for the presence of a patent ductus arteriosus or other congenital heart disease. The infant who initially improves with administration of surfactant and subsequently deteriorates should also be evaluated for nosocomial pneumonia. (20) On admission, it is appropriate to initiate antibiotic therapy in the newborn with RDS because pneumonia may present clinically in the same manner and findings on chest radiographs can be indistinguishable from RDS.

Preventing premature birth will lower the incidence of RDS. However, attempts to prevent premature births have been largely unsuccessful, with the rate of premature births still II.5\% of all births in 20I2. To benefit those infants who will deliver prematurely, multiple randomized clinical trials strongly support the use of maternal antenatal corticosteroids. Two doses of betamethasone significantly reduce the incidence of RDS, intraventricular hemorrhage, and mortality in infants age 23 to 29 weeks' gestation. (5)(39)(40)

\section{Case 4}

A 4.4 -kg female infant is delivered via caesarean section at 4I weeks' gestational age because of presumed large for gestational age status. The amniotic fluid is stained with thick meconium. She is limp and cyanotic at birth with minimal respiratory effort. Apgar scores are 2 and 7 at $\mathrm{I}$ and 5 minutes, respectively. Temperature is $99^{\circ} \mathrm{F}\left(37.2^{\circ} \mathrm{C}\right)$, pulse is $\mathrm{I} 77$ beats per minute, and respiratory rate is 80 breaths per minute. Physical examination findings are significant for marked increased work of breathing with nasal flaring, subcostal and suprasternal retractions, a barrel-shaped chest, and coarse rhonchi in bilateral lung fields. Her chest radiograph is shown in Figure I.

\section{Meconium Aspiration Syndrome}

MSAF occurs when the fetus passes meconium before birth. Infants born through MSAF are at risk for aspiration of meconium in utero or immediately after birth. Any infant who is born through MSAF and develops respiratory distress after delivery, which cannot be attributed to another cause, is diagnosed as having MAS.

Meconium is composed of lanugo, bile, vernix, pancreatic enzymes, desquamated epithelia, amniotic fluid, and mucus. Meconium is present in the gastrointestinal tract as early as 16 weeks' gestation but is not present in the lower descending colon until 34 weeks' gestation; therefore, MSAF is seldom seen in infants younger than 37 weeks' gestation. (4I) In the compromised fetus, hypoxia or acidosis may result in a peristaltic wave and relaxation of the anal sphincter, resulting in meconium passage in utero. Aspiration may occur in utero or immediately after birth as the compromised fetus gasps.

Meconium is toxic to the newborn lung, causing inflammation and epithelial injury as it migrates distally. The $\mathrm{pH}$ of meconium is 7.I to 7.2. The acidity causes airway inflammation and a chemical pneumonitis with release of cytokines. (4I) As meconium reaches the small airways, partial obstruction occurs, which results in air trapping and hyperaeration. The typical chest radiograph initially appears streaky with diffuse parenchymal infiltrates. In time, lungs become hyperinflated with patchy areas of atelectasis and infiltrate amid alveolar distension (Figure I). Surfactant is inactivated by the bile acids in meconium, resulting in localized atelectasis, so alternatively, radiographs may resemble those of RDS with low lung volumes. Although
Figure 2. Common complications of meconium aspiration syndrome include pneumothorax (left upper) and persistent pulmonary hypertension of the newborn (right upper) characterized by cyanosis with normal lung fields and decreased pulmonary vascular markings.
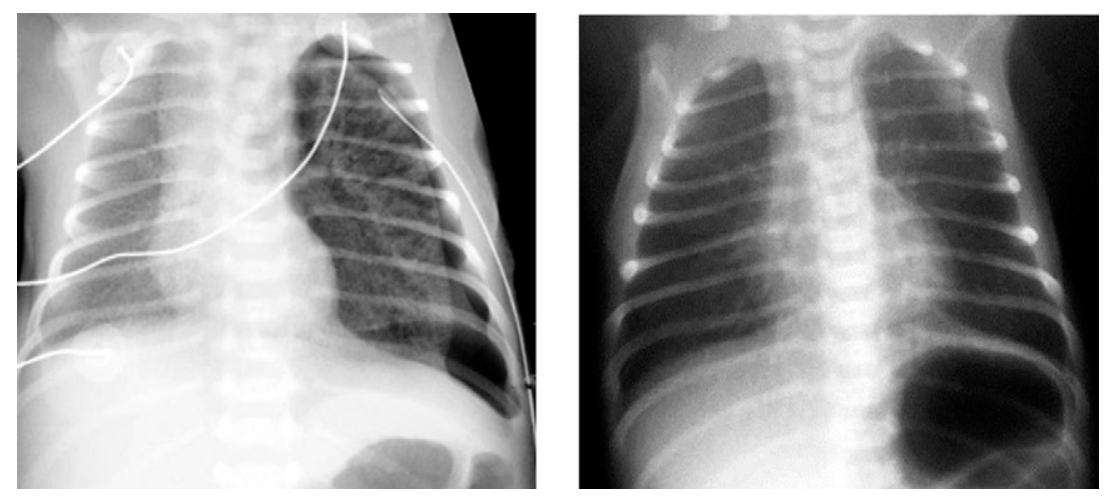
air leak syndromes may occur with other respiratory diseases of the newborn, pneumomediastinum, pneumothorax, and PPHN are common in MAS (Figure 2).

Management is directed at strategies to support the infant. Supplemental oxygen is required, and CPAP and mechanical ventilation may also be considered in severe cases. Replacement with exogenous surfactant is common practice and reduces the need for extracorporal membrane oxygenation (ECMO) and the risk of pneumothorax. (42) Because MAS results in a ventilation-perfusion mismatch whereby ventilated alveolar units are not perfused by pulmonary blood vessels, severe hypoxemia may result and further increases pulmonary vascular resistance. Echocardiography helps confirm PPHN by revealing ventricular septal wall flattening, tricuspid regurgitation, and right-toleft shunting at the patent ductus arteriosus. Inhaled nitric oxide is a selective pulmonary vasodilator without systemic effects. It is often used with high-frequency ventilation in severe cases of MAS to maintain adequate oxygenation and ventilation and reduce the need for ECMO. Initiation of broad-spectrum antibiotic therapy is appropriate because meconium is a growth medium for gram-negative organisms. Residual pulmonary compromise is common after MAS. As many as $50 \%$ of affected infants are diagnosed as having reactive airway disease during their first 6 months of life, and persistent pulmonary insufficiency is seen in children as old as 8 years. (43)

Because of the significant morbidity associated with MAS, preventive measures are important. Historically, oropharyngeal and nasopharyngeal suctioning was performed on the meconium-stained infant after delivery of the head but before delivery of the shoulders and was initially thought to be an effective preventive measure. (44) However, a large, multicenter randomized controlled trial in 2004 found that this practice does not prevent MAS or decrease the need for mechanical ventilation or hospital length of stay. (45) Consequently, routine suctioning on the perineum is no longer indicated. Endotracheal suctioning immediately after birth was also a routine practice for all meconium-stained infants until a large randomized controlled trial found that intubating and suctioning vigorous infants born through MSAF had no benefit and increased the rate of complications. (46) This finding has been confirmed by additional, well-designed studies, (47) prompting a change in practice guidelines in 2000. Current evidence still supports immediate endotracheal suctioning of the depressed infant as defined by a low heart rate (<Ioo beats per minute), poor muscle tone, and no spontaneous respiratory effort. (8) Intubation and suctioning the vigorous, spontaneously breathing infant is not recommended. (8)(47)(48)
Approximately $13 \%$ of all live births are through MSAF. Although the number of cases has decreased during the past decade, $4 \%$ to $5 \%$ of these will develop MAS. (30)(4I) Previously, many postterm infants $(\geq 42$ weeks' gestation) developed MAS. However, a recent meta-analysis provides evidence that induction of labor at $4 \mathrm{I}$ weeks' gestation reduces the risk of MAS and perinatal death without increasing the risk of caesarean section. (7) Therefore, many obstetricians do not allow pregnancies to advance beyond 4I weeks' gestation. In addition, advances in fetal heart rate monitoring have identified compromised fetuses, allowing for timely obstetric intervention that may help prevent in utero aspiration of meconium. Amnioinfusion or transcervical infusion of saline into the amniotic cavity has been proposed as a practice to decrease the incidence of MAS. Although amnioinfusion is beneficial for the distressed fetus with oligohydramnios, best evidence does not indicate a reduced risk of moderate to severe MAS or perinatal death. (49)

\section{CONCLUSION}

Learning to readily recognize respiratory distress in the newborn and understanding physiologic abnormalities associated with each of the various causes will guide optimal management. Although decreasing the incidence through preventive measures is ideal, early recognition and treatment of the common neonatal respiratory diseases will decrease both short- and long-term complications and related mortality of at-risk infants.

\section{Summary}

- Respiratory distress presents as tachypnea, nasal flaring, retractions, and grunting and may progress to respiratory failure if not readily recognized and managed.

- Causes of respiratory distress vary and may not lie within the lung. A thorough history, physical examination, and radiographic and laboratory findings will aid in the differential diagnosis. Common causes include transient tachypnea of the newborn, neonatal pneumonia, respiratory distress syndrome (RDS), and meconium aspiration syndrome (MAS).

- Strong evidence reveals an inverse relationship between gestational age and respiratory morbidity. (1)(2)(9)(25)(26) Expert opinion recommends careful consideration about elective delivery without labor at less than 39 weeks' gestation.

- Extensive evidence, including randomized control trials, cohort studies, and expert opinion, supports maternal group B streptococcus screening, intrapartum antibiotic prophylaxis, and appropriate followup of high-risk newborns according to guidelines established by the Centers for Disease Control and Prevention. (4)(29)(31)(32)(34) Following these best-practice strategies is effective in preventing neonatal pneumonia and its complications. (31)(32)(34) 
- On the basis of strong evidence, including randomized control trials and Cochrane Reviews, administration of antenatal corticosteroids (5) and postnatal surfactant (6) decrease respiratory morbidity associated with RDS.

- Trends in perinatal management strategies to prevent MAS have changed. There is strong evidence that amnioinfusion, (49) oropharyngeal and nasopharyngeal suctioning at the perineum, (45) or intubation and endotracheal suctioning of vigorous infants (46)(47) do not decrease MAS or its complications. Some research and expert opinion supports endotracheal suctioning of nonvigorous meconium-stained infants (8) and induction of labor at 41 weeks' gestation (7) to prevent MAS.

\section{References}

I. Edwards MO, Kotecha SJ, Kotecha S. Respiratory distress of the term newborn infant. Paediatr Respir Rev. 2013;I4(I):29-36

2. Hibbard JU, Wilkins I, Sun L, et al; Consortium on Safe Labor. Respiratory morbidity in late preterm births. JAMA. 2010;304 (4):4I9-425

3. Mahoney AD, Jain L. Respiratory disorders in moderately preterm, late preterm, and early term infants. Clin Perinatol. 2013;40(4): 665-678

4. Verani JR, McGee L, Schrag SJ. Division of Bacterial Diseases, National Center for Immunization and Respiratory Diseases, Centers for Disease Control and Prevention. Prevention of perinatal group B streptococcal disease: revised guidelines from CDC, 2010. MMWR Recomm Rep. 20I0;59(RR-IO):I-36

5. Carlo WA, McDonald SA, Fanaroff AA, et al; Eunice Kennedy Shriver National Institute of Child Health and Human Development Neonatal Research Network. Association of antenatal corticosteroids with mortality and neurodevelopmental outcomes among infants born at 22 to 25 weeks' gestation. JAMA. 2011;306 (2I):2348-2358

6. Bahadue FL, Soll R. Early versus delayed selective surfactant treatment for neonatal respiratory distress syndrome. Cochrane Database Syst Rev. 2012;11:CDooI456

7. Gülmezoglu AM, Crowther CA, Middleton P, Heatley E. Induction of labour for improving birth outcomes for women at or beyond term. Cochrane Database Syst Rev. 2012;6:CDoo4945

8. Bhat R, Vidyasagar D. Delivery room management of meconiumstained infant. Clin Perinatol. 2012;39(4):8I7-83I

9. Gouyon JB, Ribakovsky C, Ferdynus C, Quantin C, Sagot P, Gouyon B; Burgundy Perinatal Network. Severe respiratory disorders in term neonates. Paediatr Perinat Epidemiol. 2008;22(I):22-30

Io. Williams O, Hutchings G, Hubinont C, Debauche C, Greenough A. Pulmonary effects of prolonged oligohydramnios following midtrimester rupture of the membranes-antenatal and postnatal management. Neonatology. 2012;IOI(2):83-90

II. Piper JM, Xenakis EM, Langer O. Delayed appearance of pulmonary maturation markers is associated with poor glucose control in diabetic pregnancies. J Matern Fetal Med. I998;7(3):I48-I53

I2. Jobe AH. Effects of chorioamnionitis on the fetal lung. Clin Perinatol. 2012;39(3):44I-457
I3. Adzick NS, Harrison MR, Crombleholme TM, Flake AW, Howell LJ. Fetal lung lesions: management and outcome. Am J Obstet Gynecol. I998;I79(4):884-889

I4. Bak SY, Shin YH, Jeon JH, et al. Prognostic factors for treatment outcomes in transient tachypnea of the newborn. Pediatr Int. 2012;54(6):875-880

I5. Warren JB, Anderson JM. Newborn respiratory disorders. Pediatr Rev. 2010;3I(I2):487-495, quiz 496

I6. West JB. Respiratory Physiology: The Essentials. Baltimore, MD: Williams \& Wilkins; 2012

I7. Davis RP, Mychaliska GB. Neonatal pulmonary physiology. Semin Pediatr Surg. 2013;22(4):I79-184

I8. Wilmott RW, Boat TF, Bush A, Chernick V, Deterding RR. Kendig and Chernick's Disorders of the Respiratory Tract in Children. Philadelphia, PA: Elsevier Saunders; 2012

I9. Magder S. Bench-to-bedside review: ventilatory abnormalities in sepsis. Crit Care. 2009;13(I):202

20. Weisman LE, Hansen TN. Contemporary Diagnosis and Management of Neonatal Respiratory Diseases. 3rd ed. Newton, PA: Handbooks in Health Care Co.; 2003

2I. Bancalari E, Polin RA. The Newborn Lung: Neonatology Questions and Controversies. Philadelphia, PA: Saunders Elsevier; 2008

22. Bhandari A, McGrath-Morrow S. Long-term pulmonary outcomes of patients with bronchopulmonary dysplasia. Semin Perinatol. 2013;37(2):I32-I37

23. Aly H. Respiratory disorders in the newborn: identification and diagnosis. Pediatr Rev. 2004;25(6):20I-208

24. Elias N, O'Brodovich H. Clearance of fluid from airspaces of newborns and infants. Neoreviews. 2006;7:e88

25. Sengupta S, Carrion V, Shelton J, et al. Adverse neonatal outcomes associated with early-term birth. JAMA Pediatr. 2013;167(II): I053-1059

26. Shapiro-Mendoza CK, Tomashek KM, Kotelchuck M, et al. Effect of late-preterm birth and maternal medical conditions on newborn morbidity risk. Pediatrics. 2008;I2I(2):e223-e232

27. Jain L, Dudell GG. Respiratory transition in infants delivered by cesarean section. Semin Perinatol. 2006;30(5):296-304

28. Campbell JR. Neonatal pneumonia. Semin Respir Infect. I996;II (3): $155-162$

29. Stoll BJ, Hansen NI, Sánchez PJ, et al; Eunice Kennedy Shriver National Institute of Child Health and Human Development Neonatal Research Network. Early onset neonatal sepsis: the burden of group B streptococcal and E. coli disease continues. Pediatrics. 2OII;I27(5):8I7-826

30. Flidel-Rimon O, Shinwell ES. Respiratory distress in the term and near-term infant. Neoreviews. 2005;6:2289-e297

3I. Randis TM, Polin RA. Early-onset group B streptococcal sepsis: new recommendations from the Centres for Disease Control and Prevention. Arch Dis Child Fetal Neonatal Ed. 20I2;97(4):F29I-F294

32. Oh W. Early onset neonatal group B streptococcal sepsis. Am J Perinatol. 2013;30(2):I43-I47

33. Nissen MD. Congenital and neonatal pneumonia. Paediatr Respir Rev. 2007;8(3):195-203

34. Puopolo KM, Madoff LC, Eichenwald EC. Early-onset group B streptococcal disease in the era of maternal screening. Pediatrics. 2005;II5(5):I240-I246 
35. Madhi SA, Dangor Z, Heath PT, et al. Considerations for a phase-III trial to evaluate a group B Streptococcus polysaccharide-protein conjugate vaccine in pregnant women for the prevention of earlyand late-onset invasive disease in young-infants. Vaccine. 2013;3I (suppl 4):D52-D57

36. Dempsey AF, Pyrzanowski J, Donnelly M, et al. Acceptability of a hypothetical group B strep vaccine among pregnant and recently delivered women. Vaccine. 20I4;32(2I):2463-2468

37. Helve O, Pitkänen OM, Andersson S, O’Brodovich H, Kirjavainen T, Otulakowski G. Low expression of human epithelial sodium channel in airway epithelium of preterm infants with respiratory distress. Pediatrics. 2004;II3(5):1267-I272

38. Lista G, Castoldi F. Which clinical markers for appropriate timing of surfactant therapy?Acta Biomed. 2013;84(suppl I):I5-I7

39. Hayes EJ, Paul DA, Stahl GE, et al. Effect of antenatal corticosteroids on survival for neonates born at 23 weeks of gestation. Obstet Gynecol. 2008;III(4):92I-926

40. Abbasi S, Oxford C, Gerdes J, Sehdev H, Ludmir J. Antenatal corticosteroids prior to 24 weeks' gestation and neonatal outcome of extremely low birth weight infants. Am J Perinatol. 2010;27(I): 6I-66

4I. Yeh TF. Meconium aspiration syndrome: pathogenesis and current management. Neoreviews. 2010;11:e503-e5I
42. Findlay RD, Taeusch HW, Walther FJ. Surfactant replacement therapy for meconium aspiration syndrome. Pediatrics. I996;97(I):48-52

43. Macfarlane PI, Heaf DP. Pulmonary function in children after neonatal meconium aspiration syndrome. Arch Dis Child. I988;63 (4):368-372

44. Carson BS, Losey RW, Bowes WA Jr, Simmons MA. Combined obstetric and pediatric approach to prevent meconium aspiration syndrome. Am J Obstet Gynecol. I976;126(6):712-7I5

45. Vain NE, Szyld EG, Prudent LM, Wiswell TE, Aguilar AM, Vivas NI. Oropharyngeal and nasopharyngeal suctioning of meconiumstained neonates before delivery of their shoulders: multicentre, randomised controlled trial. Lancet. 2004;364(9434):597-602

46. Linder N, Aranda JV, Tsur M, et al. Need for endotracheal intubation and suction in meconium-stained neonates. J Pediatr. I988;II2(4):6I3-6I5

47. Wiswell TE, Gannon CM, Jacob J, et al. Delivery room management of the apparently vigorous meconium-stained neonate: results of the multicenter, international collaborative trial. Pediatrics. 2000;105 (I, pt I):I-7

48. Wiswell TE. Handling the meconium-stained infant. Semin Neonatol. 2001;6(3):225-231

49. Fraser WD, Hofmeyr J, Lede R, et al; Amnioinfusion Trial Group. Amnioinfusion for the prevention of the meconium aspiration syndrome. N Engl J Med. 2005;353(9):909-9I7 


\section{PIR Quiz}

1. Which of the following is the strongest risk factor associated with the development of transient tachypnea of the newborn (TTN)?

A. Cesarean birth of a 38-week-gestation infant.

B. Hypothermia.

C. Maternal edema.

D. Maternal preeclampsia.

E. Small for gestational age.

2. A gravida 3, para 2 mother presents at 38 weeks' gestation in spontaneous labor from home. She reports spontaneous rupture of membranes the previous day (22 hours ago) that is confirmed on inspection. She has a low-grade temperature of $100.5^{\circ} \mathrm{F}\left(38.1^{\circ} \mathrm{C}\right)$ but has not had any problems during the pregnancy and is group $B$ streptococcus negative. She is allergic to amoxicillin from which she developed a nonurticarial rash but has no other known allergies. The obstetrician asks you about the best management to prevent neonatal pneumonia. You reply:

A. Institute intrapartum antibiotic prophylaxis with intravenous cefazolin.

B. Institute intrapartum antibiotic prophylaxis with intravenous penicillin.

C. Institute intrapartum antibiotic prophylaxis with oral azithromycin.

D. Monitor fever and if it persists proceed with a caesarian section.

E. Rescreen for group B streptococcus with a nucleic acid amplification test.

3. A $3.8-\mathrm{kg}$ female infant is born to the mother presented and managed in question 2. The infant has a heart rate of 180 beats per minute and mild grunting and flaring. She is mildly pale and has oxygen saturation of $85 \%$ on room air at 20 minutes after birth. The best practice management strategy for this infant is:

A. Allow the family time to bond with the infant, placing the infant skin to skin with mother.

B. Stabilize the infant's respiratory status and observe for 48 hours.

C. Stabilize the infant's respiratory status and obtain a limited evaluation, including a complete blood cell count and blood culture.

D. Stabilize the infant's respiratory status, obtain a limited evaluation that includes a complete blood cell count and blood culture, and initiate intravenous antibiotic therapy.

E. Stabilize the infant's respiratory status, plan a complete evaluation that includes a complete blood cell count and blood and spinal fluid culture, and initiate intravenous antibiotic therapy.

4. A $1.1-\mathrm{kg}$ female is born at 29 weeks' gestation because of preterm labor. She is vigorous at birth but shows signs of significant respiratory distress evidenced by subcostal retractions, nasal flaring, and audible expiratory grunting. She is diagnosed as having respiratory distress syndrome and administered exogenous endotracheal surfactant. At what period would one expect to observe the diuretic phase of respiratory distress syndrome?
A. Within 1 day after birth.
B. 4 days after birth.
C. 7 days after birth.
D. 10 days after birth.
E. Immediately after birth.

5. A $4.5-\mathrm{kg}$ male infant is born via cesarean section at 41 weeks' gestation in meconiumstained amniotic fluid. At birth he is noted to have a low heart rate, poor muscle tone, and no spontaneous respirations. Which of the following procedures has been found to have the greatest effect on a favorable outcome in this infant?
A. Amnioinfusion 6 hours before delivery.
B. Antenatal maternal corticosteroids.
C. Endotracheal intubation and suctioning before the infant's first breath.
D. Oropharyngeal and nasopharyngeal suctioning before delivery of the shoulders.
E. Systemic macrolide antibiotic therapy.

REQUIREMENTS: Learners can take Pediatrics in Review quizzes and claim credit online only at: http://pedsinreview.org.

To successfully complete 2014 Pediatrics in Review articles for AMA PRA Category 1 Credit $^{T M}$, learners must demonstrate a minimum performance level of $60 \%$ or higher on this assessment, which measures achievement of the educational purpose and/or objectives of this activity. If you score less than $60 \%$ on the assessment, you will be given additional opportunities to answer questions until an overall $60 \%$ or greater score is achieved. 